\title{
Linear Independence of T-Spline Blending Functions of Degree One for Isogeometric Analysis
}

\author{
Aizeng Wang ${ }^{1,2,3, *}$, Ling Li ${ }^{1}$, Wei Wang ${ }^{1}$, Xiaoxiao Du ${ }^{1}$, Feng Xiao ${ }^{1}$, Zhanchuan Cai ${ }^{2}$ and Gang Zhao ${ }^{1,3, *}$ \\ 1 School of Mechanical Engineering \& Automation, Beihang University, Beijing 100191, China; \\ linglee@buaa.edu.cn (L.L.); jrr@@buaa.edu.cn (W.W.); duxxhf@163.com (X.D.); xiaoyaoyou@buaa.edu.cn (F.X.) \\ 2 State Key Laboratory of Lunar and Planetary Sciences, Macau University of Science and Technology, \\ Macau 999078, China; zccai@must.edu.mo \\ 3 State Key Laboratory of Virtual Reality Technology \& Systems, Beihang University, Beijing 100191, China \\ * Correspondence: azwang@buaa.edu.cn (A.W.); zhaog@buaa.edu.cn (G.Z.)
}

check for updates

Citation: Wang, A.; Li, L.; Wang, W.; Du, X.; Xiao, F.; Cai, Z.; Zhao, G. Linear Independence of T-Spline Blending Functions of Degree One for Isogeometric Analysis. Mathematics 2021, 9, 1346. https://doi.org/ $10.3390 /$ math 9121346

Academic Editor: Jean-Charles Pinoli

Received: 11 May 2021

Accepted: 8 June 2021

Published: 10 June 2021

Publisher's Note: MDPI stays neutral with regard to jurisdictional claims in published maps and institutional affiliations.

Copyright: (c) 2021 by the authors. Licensee MDPI, Basel, Switzerland. This article is an open access article distributed under the terms and conditions of the Creative Commons Attribution (CC BY) license (https:/ / creativecommons.org/licenses/by/ $4.0 /)$.

\begin{abstract}
Linear independence of the blending functions is a necessary requirement for T-spline in isogeometric analysis. The main work in this paper focuses on the analysis about T-splines of degree one, we demonstrate that all the blending functions of such T-spline of degree one are linearly independent. The advantage owned by one degree T-spline is that it can avoid the problem of judging whether the model is analysis-suitable or not, especially for occasions that need a quick response from the analysis results. This may provide a new way of using T-spline for a CAD and CAE integrating scenario, since one degree T-spline still guarantees the topology flexibility and is compatible with the spline-based modeling system. In addition, we compare the numerical approximations of isogeometric analysis and finite element analysis, and the experiment indicates that isogeometric analysis using T-spline of degree one can reach a comparable result with classical method.
\end{abstract}

Keywords: T-splines; CAD/CAE; finite element analysis; isogeometric analysis

\section{Introduction}

Finite element analysis (FEA), which has a wide range of applications in industry, is a classic numerical simulation, but during the analysis progress the spline model generated by computer-aided design (CAD) needs to discretize into a computer-aided engineering (CAE) mesh model. This translation is highly time consuming, which affects the accuracy seriously. In order to solve this problem, isogeometric analysis (IGA) was proposed by Hughes et al. in 2005 as a new analysis technology for computer-aided engineering [1]. IGA utilizes spline functions as a basis for the integration of CAD and CAE [1-4], and the development of T-splines accelerates the spline-based isogeometric analysis [5-8]. For IGA, linear independence of blending functions is a necessary requirement, since the equation system is invertible.

In 2010, Buffa et al. gave an example of a T-spline with linearly dependent blending functions [9]. That is to say not all T-splines are suitable for IGA. Later, in 2011, the authors presented an algorithm to check whether blending functions of a given T-spline are linearly independent or not [10]. However, the algorithm needs more computation, since local refinement operations are included. In 2012, Li et al. gave analysis-suitable T-splines (AST), whose blending functions are linearly independent, and visualized the linearly independence on the topology [11]. However, the topology condition is strict because L- and I-junctions are not allowed. In order to get rid of this limitation and extend the scope of the models for IGA, Li et al. proposed AS++ T-spline [12] and proved the linear independence from the perspective of dual basis. Although IGA based on T-spline had a booming development in thermodynamics [13], aerodynamics [14,15], nanometer materials [16,17], or the estimate of stability [18] and reliability [19], AST cannot satisfy 
the application requirement for complex models. Like FEA, the linear independence of blending functions is a precondition in IGA, and even spline wavelets transform [20].

The original intention to use T-spline instead of non-uniform rational B-spline (NURBS) in IGA was that T-spline owns certain properties over NURBS, such as local refinement and watertightness $[5,6,11]$. While a higher degree of T-splines may be more accurate in simulation, the independence is so complicated to assure. In the meantime, we could conduct a research on lower T-spline. On the one hand, T-spline of degree one owns the impressive advantages of T-spline and, on the other hand, it could also achieve higher accuracy with enough knots.

Although other basic functions have been introduced by Hughes [21], they are not equal with our work. The shape function in the literature [21] is constructed by products of linear function or Lagrange polynomials, while our blending functions are constructed by T-splines in this paper. The most essential difference is that the linear functions in the literature [21] are used for finite element analysis, and our T-spline of degree one is applied to IGA. These two various analysis methods result in diverse numerical simulations, and what is more, due to the exerting of T-spline the topological structure is not restricted by rectangular topology, and T-, L- and I-junctions are contained, as demonstrated in Figure 1.

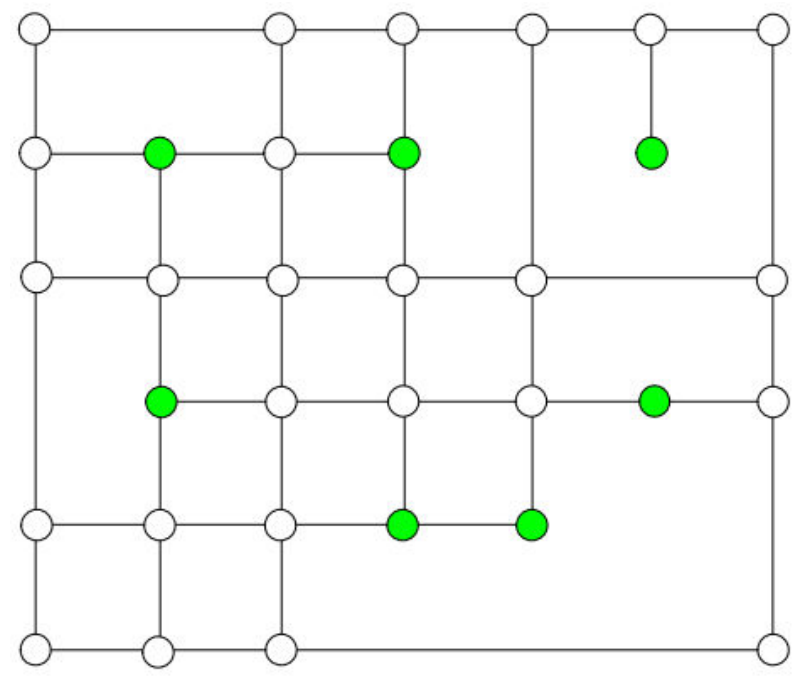

Figure 1. Topological structure of a T-mesh.

So, we make a further exploration about T-splines of degree one to generalize the scope of linear independence, which contains T-, L- and I-junctions. The main results of this paper can be summarized as follows: Firstly, we prove that the blending functions for any T-spline of degree one are linearly independent. Secondly, this result provides a mathematical foundation of one degree T-splines for isogeometric analysis, because it can avoid the problem of judging whether it is analysis-suitable or not. This may provide a new way of using T-spline for a CAD and CAE integrating scenario, since one degree T-spline guarantees topology flexibility and is compatible with NURBS-based system.

The structure of this paper is organized as follows: Theoretical demonstration is shown in Section 2. Section 3 presents some examples of degree one, and a suitable model is exhibited based on IGA and FEA to validate the results in Section 4. At the end of this paper, we give a brief conclusion and future work.

\section{Linear Independence of Blending Functions of Degree One}

\subsection{B-Spline Functions of Degree One}

Suppose the following:

$$
N_{0,1}(u)=\sum_{i=1}^{n} a_{i} N_{i, 1}(u) \quad\left(a_{i} \neq 0\right)
$$


where $N_{i, 1}(u)$ is a B-spline function of degree one associated with knots $\left\{u_{-1}^{i}, u_{0}^{i}, u_{1}^{i}\right\}$ $\left(u_{-1}^{i}<u_{0}^{i}<u_{1}^{i}\right)$, and $\left\{N_{i, 1}(u) \mid 1 \leq i \leq n\right\}$ are linearly independent. Equation (1) can be rewritten as follows:

$$
\sum_{j=0}^{m} k_{j} N_{j, 1}(u)=\sum_{j=m+1}^{n} k_{j} N_{j, 1}(u)
$$

where $k_{j}>0(0 \leq j \leq n)$.

$$
\text { Let } \Xi_{u}^{0}=\left\{u_{0}^{j} \mid 0 \leq j \leq m\right\} \text {. }
$$

Proposition 1. If $\sum_{j=0}^{m} k_{j} N_{j, 1}(u)\left(k_{j}>0\right)$ achieves a local maximum value at $u=u^{*}$, then $u^{*} \in \Xi_{u}^{0}$.

Proof. For $u=u^{*} \notin \Xi_{u}^{0},\left\{N_{j, 1}(u) \mid 0 \leq j \leq m\right\}$ can be divided into the following two parts: $Z=\left\{N_{z, 1}(u) \mid N_{z, 1}\left(u^{*}\right)=0\right\}$ and $T=\left\{N_{t, 1}(u) \mid N_{t, 1}\left(u^{*}\right) \neq 0\right\}$. In other words, $Z$ is a set of functions $\left\{N_{z, 1}(u) \mid N_{z, 1}\left(u^{*}\right)=0\right\}$, and $T$ is a set of functions $\left\{N_{t, 1}(u) \mid N_{t, 1}\left(u^{*}\right) \neq 0\right\}$, where $Z \cap T=\phi$.

Let $f(u)=\sum_{j=0}^{m} k_{j} N_{j, 1}(u)\left(k_{j}>0\right)$. If $u=u^{*} \notin \Xi_{u}^{0}$, the left and the right derivatives of $f(u)$ at $u=u^{*}$ are $f_{-}^{\prime}=k_{T}+k_{\alpha}$ and $f_{+}^{\prime}=k_{T}+k_{\beta}$, respectively, where $k_{T} \quad=\quad\left(\sum_{t} k_{t} N_{t, 1}\left(u^{*}\right) \mid N_{t, 1}(u) \in T\right)^{\prime}, \quad k_{\alpha}=\left(\sum_{z} k_{z} N_{z, 1}\left(u^{*}\right) \mid N_{z, 1}(u) \in Z\right)^{\prime}$, $k_{\beta}=\left(\sum_{z} k_{z} N_{z, 1}\left(u^{*}\right) \mid N_{z, 1}(u) \in Z\right)_{+}^{\prime}$. (a) For $T=\left\{N_{t, 1}(u) \mid N_{t, 1}\left(u^{*}\right) \neq 0\right\}$, if $u^{*} \notin \Xi_{u}^{0}$, we can infer that the left derivative of $N_{t, 1}(u) \in T$ at $u=u^{*}$ is equal to the right one. That is, $k_{T}=$ $\left(\sum_{t} k_{t} N_{t, 1}\left(u^{*}\right) \mid N_{t, 1}(u) \in T\right)^{\prime}=\left(\sum_{t} k_{t} N_{t, 1}\left(u^{*}\right) \mid N_{t, 1}(u) \in T\right)_{-}^{\prime}=\left(\sum_{t} k_{t} N_{t, 1}\left(u^{*}\right) \mid N_{t, 1}(u) \in T\right)_{+}^{\prime} ;$ (b) For $Z=\left\{N_{z, 1}(u) \mid N_{z, 1}\left(u^{*}\right)=0\right\}$, if $u^{*} \notin \Xi_{u}^{0}$, we can understand that the left derivative of $N_{z, 1}(u) \in Z$ at $u=u^{*}$ is not equal to the right one. That is, $k_{\alpha}=\left(\sum_{z} k_{z} N_{z, 1}\left(u^{*}\right) \mid N_{z, 1}(u) \in Z\right)_{-}^{\prime} \neq$ $k_{\beta}=\left(\sum_{z} k_{z} N_{z, 1}\left(u^{*}\right) \mid N_{z, 1}(u) \in Z\right)_{+}^{\prime}$. From (a) and (b), we can infer that $f_{-}^{\prime}=k_{T}+k_{\alpha}$ and $f_{+}^{\prime}=k_{T}+k_{\beta}$ at $u=u^{*}$.

Because $u=u^{*} \notin \Xi_{u}^{0}$ and $N_{z, 1}\left(u^{*}\right)=0$, the left derivative of $N_{z, 1}(u)$ at $u=u^{*}$, is less than or equal to zero. That is, the following:

$$
\left(N_{z, 1}\left(u^{*}\right) \mid N_{z, 1}(u) \in Z\right)_{-}^{\prime} \leq 0
$$

From Equation (3), we get the following:

$$
k_{\alpha}=\left(\sum_{z} k_{z} N_{z, 1}\left(u^{*}\right) \mid N_{z, 1}(u) \in Z\right)_{-}^{\prime} \leq 0\left(k_{z}>0\right)
$$

Similarly, the following applies:

$$
k_{\beta}=\left(\sum_{z} k_{z} N_{z, 1}\left(u^{*}\right) \mid N_{z, 1}(u) \in Z\right)_{+}^{\prime} \geq 0\left(k_{z}>0\right)
$$

From Equations (4) and (5), we get the following:

$$
k_{\beta}-k_{\alpha} \geq 0
$$


Because $f(u)$ achieves the local maximum value at $u=u^{*}$, we get $f_{-}^{\prime}>f_{+}^{\prime}$ at $u=u^{*}$. If $k_{\beta}-k_{\alpha}=0$, we get $f_{-}^{\prime}=f_{+}^{\prime}$ at $u=u^{*}$. It is a contradiction. That is, the following:

$$
k_{\beta}-k_{\alpha}>0
$$

From Equation (7), the following can be inferred:

(case 1) if $f_{-}^{\prime}=k_{T}+k_{\alpha} \geq 0$, then $f_{+}^{\prime}=k_{T}+k_{\beta}>0$;

(case 2) if $f_{-}^{\prime}=k_{T}+k_{\alpha}<0$, then $f_{+}^{\prime}>0$ or $f_{+}^{\prime} \leq 0$.

If $f(u)$ achieves the local maximum value at $u=u^{*}, f_{+}^{\prime}\left(u^{*}\right)$ must be less than or equal to zero, and $f_{-}^{\prime}\left(u^{*}\right)$ must be bigger than or equal to zero. Based on case 1 and case 2 , the result of Proposition 1 can be obtained.

Theorem 1. For Equation (2), there exists $u_{0}^{i_{0}}=u_{0}^{j_{0}} \in \Xi_{u}^{0}$, where $i_{0} \neq j_{0}, 0 \leq i_{0} \leq m$ and $m+1 \leq j_{0} \leq n$.

Proof. From the result of Proposition 1, suppose $\sum_{j=0}^{m} k_{j} N_{j, 1}(u)\left(k_{j}>0\right)$ achieve a local maximum value at $u=u_{0}^{i_{0}} \in \Xi_{u}^{0}$, then $\sum_{j=m+1}^{n} k_{j} N_{j, 1}(u)\left(k_{j}>0\right)$ must achieve the same local maximum value at $u=u_{0}^{j_{0}}$. That is, there exists $u_{0}^{i_{0}}=u_{0}^{j_{0}} \in \Xi_{u}^{0}$, where $0 \leq i_{0} \leq m$, and $m+1 \leq j_{0} \leq n$.

\subsection{Blending Functions of Degree One}

Suppose the following:

$$
T_{0,1}(u, v)=\sum_{i=1}^{n} a_{i} T_{i, 1}(u, v)\left(a_{i} \neq 0\right)
$$

where $T_{i, 1}(u, v)$ represents a blending function of degree one, and $\left\{T_{i, 1}(u, v) \mid 1 \leq i \leq n\right\}$ are linearly independent. Let the following apply:

$$
\begin{aligned}
T_{i, 1}(u, v) & =N_{i, 1}\left[\mathbf{u}_{i}\right] N_{i, 1}\left[\mathbf{v}_{i}\right](0 \leq i \leq n) \\
\mathbf{u}_{i} & =\left[u_{-1}^{i}, u_{0}^{i}, u_{+1}^{i}\right] \\
\mathbf{v}_{i} & =\left[v_{-1}^{i}, v_{0}^{i}, v_{+1}^{i}\right]
\end{aligned}
$$

where $u_{-1}^{i}<u_{0}^{i}<u_{1}^{i}$ and $v_{-1}^{i}<v_{0}^{i}<v_{1}^{i}$. Then, Equation (8) can be rewritten as follows:

$$
\sum_{j=0}^{m} k_{j} N_{j, 1}(u) N_{j, 1}(v)=\sum_{j=m+1}^{n} k_{j} N_{j, 1}(u) N_{j, 1}(v)\left(k_{j}>0\right)
$$

Let $\Xi_{u}^{0}=\left\{u_{0}^{j} \mid 0 \leq j \leq m\right\}, \Xi_{v}^{0}=\left\{v_{0}^{j} \mid 0 \leq j \leq m\right\}$.

Suppose $T_{j, 1}(u, v)=N_{j, 1}\left[\mathbf{u}_{j}\right] N_{j, 1}\left[\mathbf{v}_{j}\right](0 \leq j \leq m)$ is defined on a T-mesh (see Figure 2a). Let the following apply:

$$
E=\left\{\overline{\left(u_{-1}^{j}, v_{0}^{j}\right)\left(u_{0}^{j}, v_{0}^{j}\right)}, \overline{\left(u_{0}^{j}, v_{0}^{j}\right)\left(u_{+1}^{j}, v_{0}^{j}\right)}, \overline{\left(u_{0}^{j}, v_{-1}^{j}\right)\left(u_{0}^{j}, v_{0}^{j}\right)}, \overline{\left(u_{0}^{j}, v_{0}^{j}\right)\left(u_{0}^{j}, v_{+1}^{j}\right)} \mid 0 \leq j \leq m\right\}
$$

where $E$ represents a set of line segments, which associate with $\mathbf{u}_{j}$ and $\mathbf{v}_{j}(0 \leq j \leq m)$. If a line segment $e \in E$ is not an edge on the T-mesh, then the line segment is called as an extension edge (see Figure $2 b$ ). 


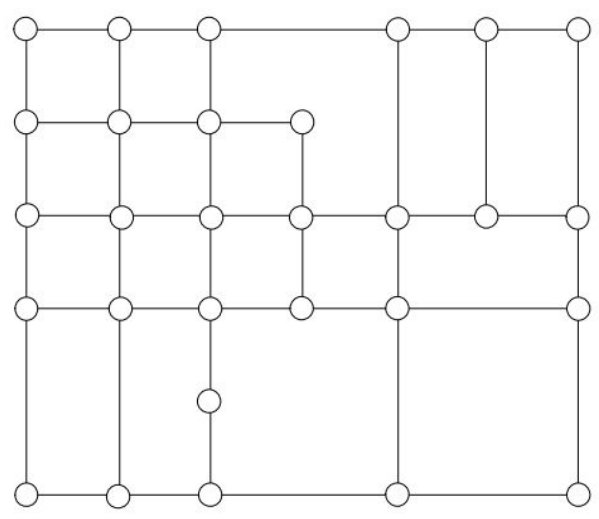

(a) T-mesh

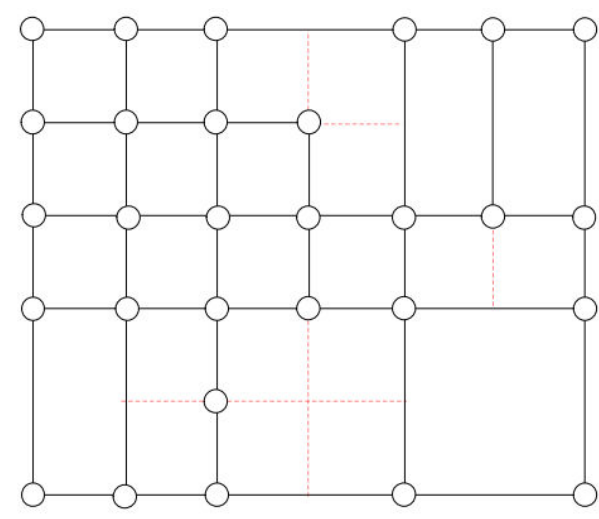

(b) Extension edges

Figure 2. Extension edges of a T-mesh. (a) T-mesh; (b) extension edges: the red dashed line segments.

If a horizontal extension edge intersects a vertical extension edge at $\left(u_{0}^{i_{0}}, v_{0}^{j_{0}}\right)$, this point is called an extension point (see Figure 3). It can be inferred that $i_{0} \neq j_{0}$ for any extension point.

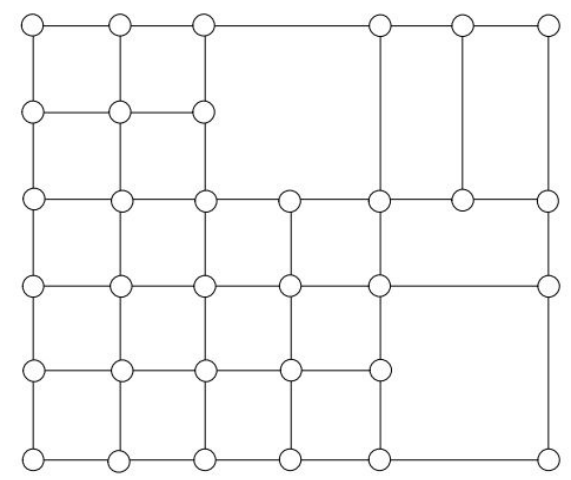

(a) T-mesh

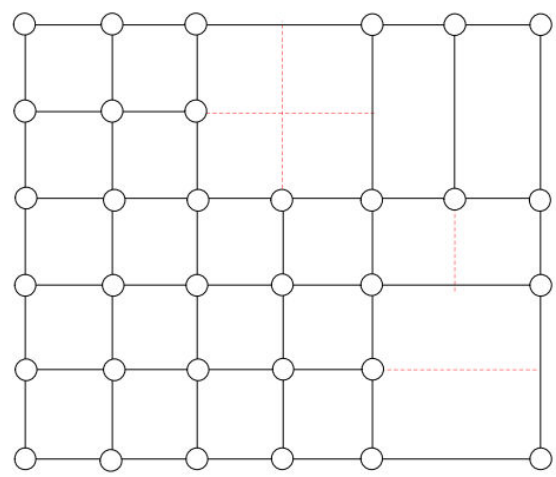

(b) Extension edges

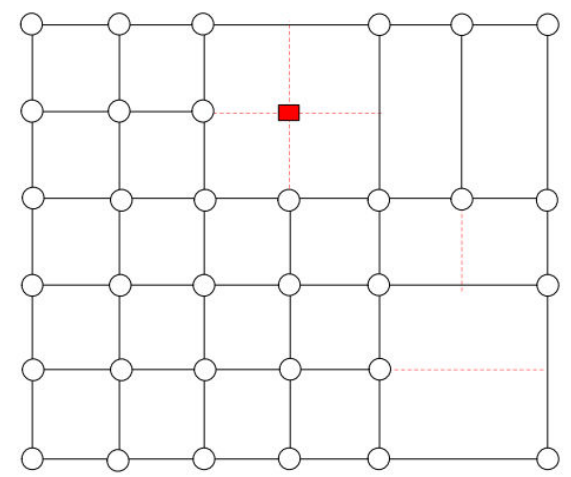

(c) Extension point

Figure 3. Extension edges and the corresponding extension point.

Proposition 2. If $\sum_{j=0}^{m} k_{j} N_{j, 1}(u) N_{j, 1}(v)\left(k_{j}>0\right)$ achieves a local maximum value at $(u, v)=$ $\left(u^{*}, v^{*}\right)$, then $\left(u^{*}, v^{*}\right) \in \Xi_{u}^{0,\left(u^{*}, v^{*}\right)} \times \Xi_{v}^{0,\left(u^{*}, v^{*}\right)}$.

Proof. Let $f(u, v)=\sum_{j=0}^{m} k_{j} N_{j, 1}(u) N_{j, 1}(v)\left(k_{j}>0\right)$, based on the given conditions, we get (a) $f\left(u, v^{*}\right)$ achieves the local maximum at $u=u^{*}$ along $u$ direction; (b) $f\left(u^{*}, v\right)$ also achieves the local maximum at $v=v^{*}$ along $\mathrm{v}$ direction. $f\left(u, v^{*}\right)$ and $f\left(u^{*}, v\right)$ can be represented as follows:

$$
\begin{aligned}
& f\left(u, v^{*}\right)=\sum_{j=0}^{m} k_{j} N_{j, 1}(u) N_{j, 1}\left(v^{*}\right)\left(k_{j}>0\right) \\
& f\left(u^{*}, v\right)=\sum_{j=0}^{m} k_{j} N_{j, 1}\left(u^{*}\right) N_{j, 1}(v)\left(k_{j}>0\right)
\end{aligned}
$$

Let

$$
\begin{aligned}
& \Xi_{u}^{0,\left(u^{*}, v^{*}\right)}=\left\{u_{0}^{j} \mid 0 \leq j \leq m, N_{j, 1}\left(v^{*}\right) \neq 0\right\} \\
& \Xi_{v}^{0,\left(u^{*}, v^{*}\right)}=\left\{v_{0}^{j} \mid 0 \leq j \leq m, N_{j, 1}\left(u^{*}\right) \neq 0\right\}
\end{aligned}
$$


It can be inferred that $\Xi_{u}^{0,\left(u^{*}, v^{*}\right)} \subset \Xi_{u}^{0}$ and $\Xi_{v}^{0,\left(u^{*}, v^{*}\right)} \subset \Xi_{v}^{0}$.

From Equations (14) and (16), we get $u^{*} \in \Xi_{u}^{0,\left(u^{*}, v^{*}\right)}$ according to Proposition 1. Similarly, we get $v^{*} \in \Xi_{v}^{0,\left(u^{*}, v^{*}\right)}$ from Equations (15) and (17). That is, $\left(u^{*}, v^{*}\right) \in \Xi_{u}^{0,\left(u^{*}, v^{*}\right)} \times$ $\Xi_{v}^{0,\left(u^{*}, v^{*}\right)}$

For $\left(u^{*}, v^{*}\right) \in \Xi_{u}^{0,\left(u^{*}, v^{*}\right)} \times \Xi_{v}^{0,\left(u^{*}, v^{*}\right)}$, the possible topology can be classified as follows: (case 1): $\left(u^{*}, v^{*}\right) \in \Xi_{u, v}^{0}$

where $\Xi_{u, v}^{0}=\left\{\left(u_{0}^{j}, v_{0}^{j}\right)\right\}, \Xi_{u, v}^{0}$ is corresponding to a set of control points (see Figure 4a). If $\left(u^{*}, v^{*}\right)=\left(u_{0}^{j_{0}}, v_{0}^{j_{0}}\right) \in \Xi_{u, v}^{0}$, it is obvious that $u_{0}^{j_{0}} \in \Xi_{u}^{0,\left(u_{0}^{j_{0}}, v_{0}^{j_{0}}\right)}, v_{0}^{j_{0}} \in \Xi_{v}^{0,\left(u_{0}^{j_{0}}, v_{0}^{j_{0}}\right)}$.

(case 2$):\left(u^{*}, v^{*}\right) \in \Xi_{u, v}^{e}$ where $\Xi_{u, v}^{e}=\left\{\left(u_{0}^{i}, v_{0}^{j}\right)\right\}$ is a set that contains the corresponding extension points (see Figure $4 \mathrm{~b})$. If $\left(u^{*}, v^{*}\right)=\left(u_{0}^{i_{0}}, v_{0}^{j_{0}}\right) \in \Xi_{u, v}^{e}$, we get $u_{0}^{i_{0}} \in \Xi_{u}^{0,\left(u_{0}^{i_{0}}, v_{0}^{j_{0}}\right)}, v_{0}^{j_{0}} \in \Xi_{v}^{0,\left(u_{0}^{i_{0}}, v_{0}^{j_{0}}\right)}$.

(case 3): $\left(u^{*}, v^{*}\right) \in \Xi_{u, v}^{z}$ where $\Xi_{u, v}^{z}$ is corresponding to a set of triangle points (see Figure 4c), which does not contain the control points and extension points.

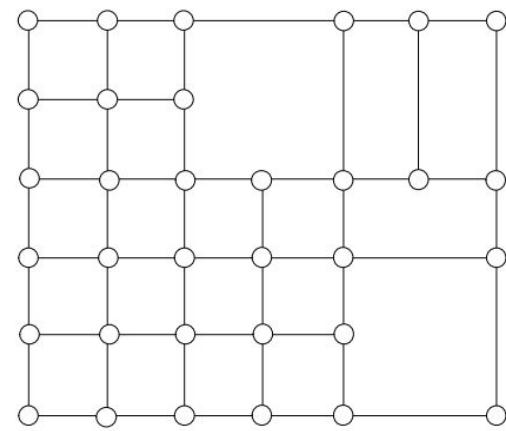

(a) $\Xi_{u, v}^{0}$ : control points

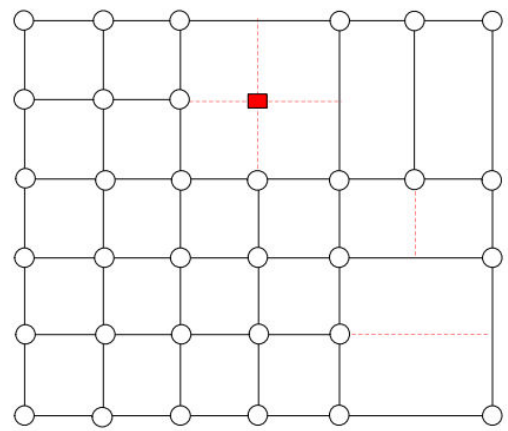

(b) $\Xi_{u, v}^{e}$ : extension points

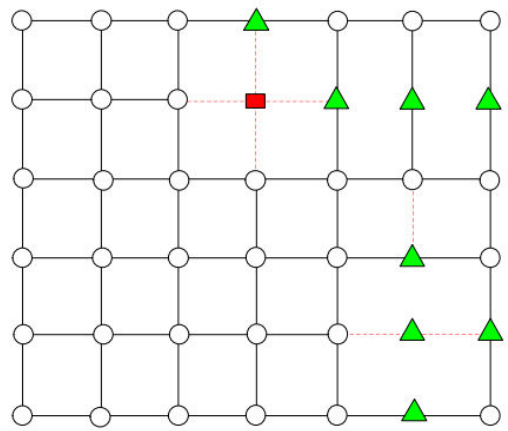

(c) $\Xi_{u, v}^{z}:$ triangle points

Figure 4. Possible topology of $\left(u^{*}, v^{*}\right) \in \Xi_{u}^{0,\left(u^{*}, v^{*}\right)} \times \Xi_{v}^{0,\left(u^{*}, v^{*}\right)}$.

Lemma 1. $\left(u^{*}, v^{*}\right) \notin \Xi_{u, v}^{z}$

Proof. According to Proposition 2, we obtain $u^{*}=u_{0}^{i_{0}} \in \Xi_{u}^{0,\left(u^{*}, v^{*}\right)}$ and $v^{*}=v_{0}^{j_{0}} \in \Xi_{v}^{0,\left(u^{*}, v^{*}\right)}$. If $\left(u^{*}, v^{*}\right) \in \Xi_{u, v}^{z}$ (see Figure $4 \mathrm{c}$ ), we get $N_{j, 1}\left(u^{*}\right)=0$ or $N_{j, 1}\left(v^{*}\right)=0$ for $0 \leq j \leq m$. That is, $\Xi_{u}^{0,\left(u^{*}, v^{*}\right)}=\phi$ or $\Xi_{v}^{0,\left(u^{*}, v^{*}\right)}=\phi$. It is a contradiction. That is, $\left(u^{*}, v^{*}\right) \notin \Xi_{u, v}^{z}$.

Lemma 2. $\left(u^{*}, v^{*}\right) \in \Xi_{u, v}^{0} \cup \Xi_{u, v}^{e}$

Proof. From Proposition 2 and Lemma 1, we get (1) when $i_{0}=j_{0},\left(u^{*}, v^{*}\right)=\left(u_{0}^{i_{0}}, v_{0}^{j_{0}}\right) \in$ $\Xi_{u, v}^{0} ;(2)$ when $i_{0} \neq j_{0},\left(u^{*}, v^{*}\right)=\left(u_{0}^{i_{0}}, v_{0}^{j_{0}}\right) \in \Xi_{u, v}^{e}$. That is, $\left(u^{*}, v^{*}\right) \in \Xi_{u, v}^{0} \cup \Xi_{u, v}^{e}$, the result of Lemma 2 is obtained.

Theorem 2. For Equation (12), there exists $\left(u_{0}^{i_{0}}, v_{0}^{j_{0}}\right)=\left(u_{0}^{i_{1}}, v_{0}^{j_{1}}\right) \in \Xi_{u, v}^{0} \cup \Xi_{u, v}^{e}$, where $\left\{\begin{array}{l}0 \leq i_{0} \leq m \\ 0 \leq j_{0} \leq m\end{array}\right.$ and $\left\{\begin{array}{l}m+1 \leq i_{1} \leq n \\ m+1 \leq j_{1} \leq n\end{array}\right.$. 
Proof. From the result of Lemma 2, suppose $\sum_{j=0}^{m} k_{j} N_{j, 1}(u) N_{j, 1}(v)\left(k_{j}>0\right)$ achieves a local maximum value at $(u, v)=\left(u_{0}^{i_{0}}, v_{0}^{j_{0}}\right) \in \Xi_{u, v}^{0} \cup \Xi_{u, v}^{e}$, then $\sum_{j=m+1}^{n} k_{j} N_{j, 1}(u) N_{j, 1}(v)\left(k_{j}>0\right)$ must achieve the same local maximum value at $(u, v)=\left(u_{0}^{i_{1}}, v_{0}^{j_{1}}\right)$. That is, $\left(u_{0}^{i_{0}}, v_{0}^{j_{0}}\right)=$ $\left(u_{0}^{i_{1}}, v_{0}^{j_{1}}\right) \in \Xi_{u, v}^{0} \cup \Xi_{u, v}^{e}$, where $\left\{\begin{array}{l}0 \leq i_{0} \leq m \\ 0 \leq j_{0} \leq m\end{array},\left\{\begin{array}{l}m+1 \leq i_{1} \leq n \\ m+1 \leq j_{1} \leq n\end{array}\right.\right.$.

Theorem 3. For any T-spline of degree one, its blending functions are linearly independent.

Proof. From Theorem 2, if the blending functions of degree one are linearly dependent, there exists $\left(u_{0}^{i_{0}}, v_{0}^{j_{0}}\right)=\left(u_{0}^{i_{1}}, v_{0}^{j_{1}}\right) \in \Xi_{u, v}^{0} \cup \Xi_{u, v}^{e}$, where $0 \leq i_{0} \leq m, 0 \leq j_{0} \leq m ; m+1 \leq$ $i_{1} \leq n, m+1 \leq j_{1} \leq n$. For $\left(u_{0}^{i_{0}}, v_{0}^{j_{0}}\right)=\left(u_{0}^{i_{1}}, v_{0}^{j_{1}}\right) \in \Xi_{u, v}^{0} \cup \Xi_{u, v}^{e}$, the possible topology can be classified as follows:

(case 1): $i_{0}=j_{0}, i_{1}=j_{1}$

That is, $\left(u_{0}^{i_{0}}, v_{0}^{j_{0}}\right)=\left(u_{0}^{i_{1}}, v_{0}^{j_{1}}\right) \in \Xi_{u, v}^{0} .\left(u_{0}^{i_{0}}, v_{0}^{j_{0}}\right)$ and $\left(u_{0}^{i_{1}}, v_{0}^{j_{1}}\right)$ correspond to a same control point on a T-mesh without multiple knots. That is $T_{i_{0}, 1}(u, v)=T_{j_{0}, 1}(u, v)$. It is a contradiction.

(case 2): $i_{0}=j_{0}, i_{1} \neq j_{1}$

For this case, $\left(u_{0}^{i_{0}}, v_{0}^{j_{0}}\right) \in \Xi_{u, v}^{0},\left(u_{0}^{i_{1}}, v_{0}^{j_{1}}\right) \in \Xi_{u, v}^{e} .\left(u_{0}^{i_{0}}, v_{0}^{j_{0}}\right)=\left(u_{0}^{i_{1}}, v_{0}^{j_{1}}\right),\left(u_{0}^{i_{0}}, v_{0}^{j_{0}}\right)$ and $\left(u_{0}^{i_{1}}, v_{0}^{j_{1}}\right)$ correspond to a control point and an extension point, respectively. It is a contradiction for a T-mesh without multiple knots.

(case 3): $i_{0} \neq j_{0}, i_{1}=j_{1}$

Similar to case 2 , it is also a contradiction.

(case 4$): i_{0} \neq j_{0}, i_{1} \neq j_{1}$

For this case, $\left(u_{0}^{i_{0}}, v_{0}^{j_{0}}\right) \in \Xi_{u, v}^{e},\left(u_{0}^{i_{1}}, v_{0}^{j_{1}}\right) \in \Xi_{u, v}^{e} \cdot\left(u_{0}^{i_{0}}, v_{0}^{j_{0}}\right)=\left(u_{0}^{i_{1}}, v_{0}^{j_{1}}\right),\left(u_{0}^{i_{0}}, v_{0}^{j_{0}}\right)$ and $\left(u_{0}^{i_{1}}, v_{0}^{j_{1}}\right)$ correspond to a same extension point (see Figure 5). However, this topology is not allowed for T-meshes [5,6].

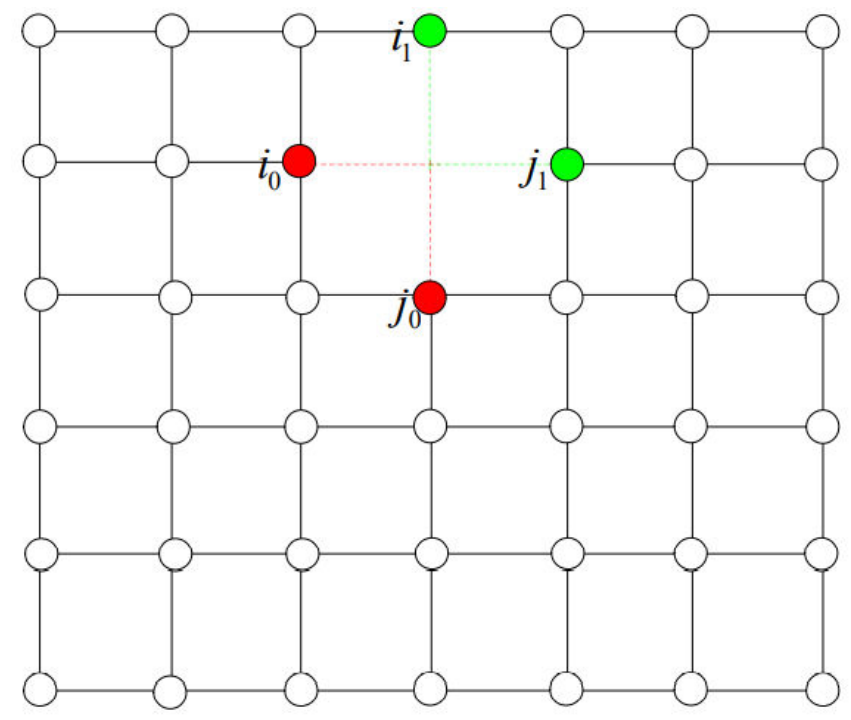

Figure 5. Possible topology of case 4 . 


\section{Examples of One Degree T-Splines}

In this section, we give three examples of linearly independent T-splines of degree one. In each of these examples, a detailed explanation is provided.

Example 1. The first example is a T-mesh with knot vectors $[0,1 / 7,2 / 7,3 / 7,4 / 7,5 / 7,6 / 7,1]$ and $[0,1 / 6,2 / 6,3 / 6,4 / 6,5 / 6,1]$ along $u$ and $v$ directions (see Figure 6 ). From Theorem 3 , the blending functions of degree one, which are defined on the T-mesh, are linearly independent.

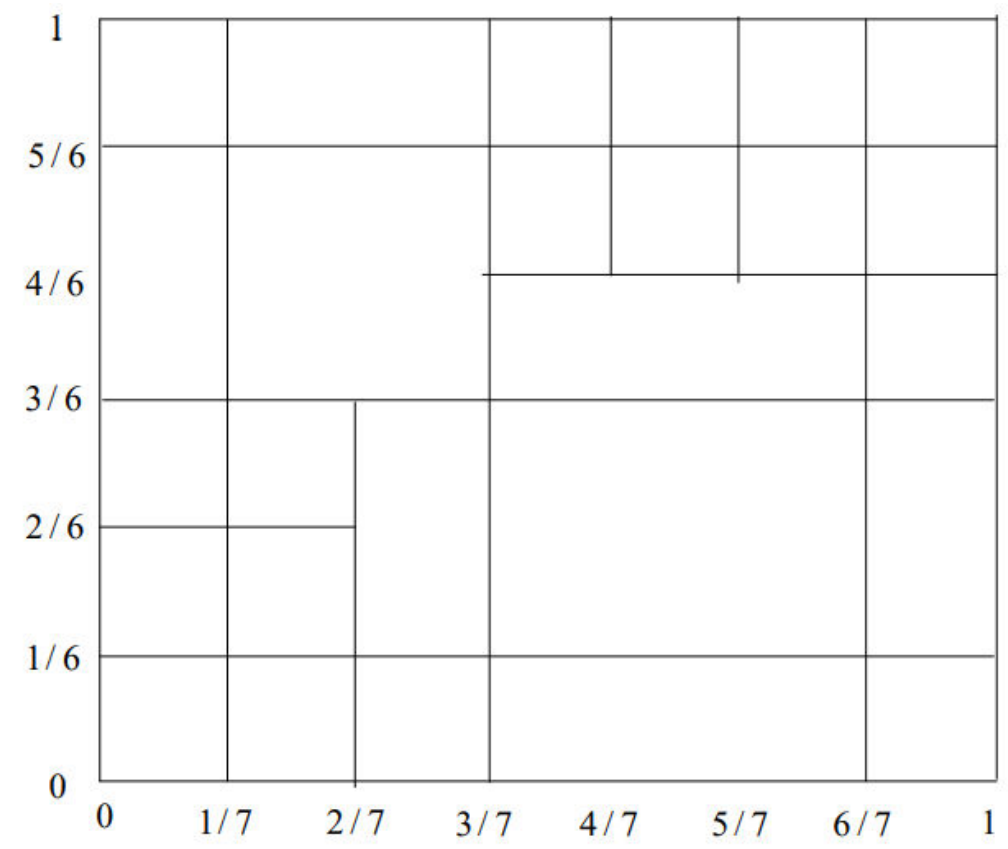

Figure 6. The first example.

Example 2. The second example is a T-mesh with knot vectors $\left[u_{0}, u_{1}, u_{2}, u_{3}, u_{4}, u_{5}, u_{6}, u_{7}\right]$ $\left(u_{i} \neq u_{j}\right.$, for $\left.i \neq j\right)$ and $\left[v_{0}, v_{1}, v_{2}, v_{3}, v_{4}, v_{5}, v_{6}\right]\left(v_{i} \neq v_{j}\right.$, for $\left.i \neq j\right)$ along $u$ and $v$ directions (see Figure 7). The blending functions of degree one associated with the T-mesh are linearly independent.

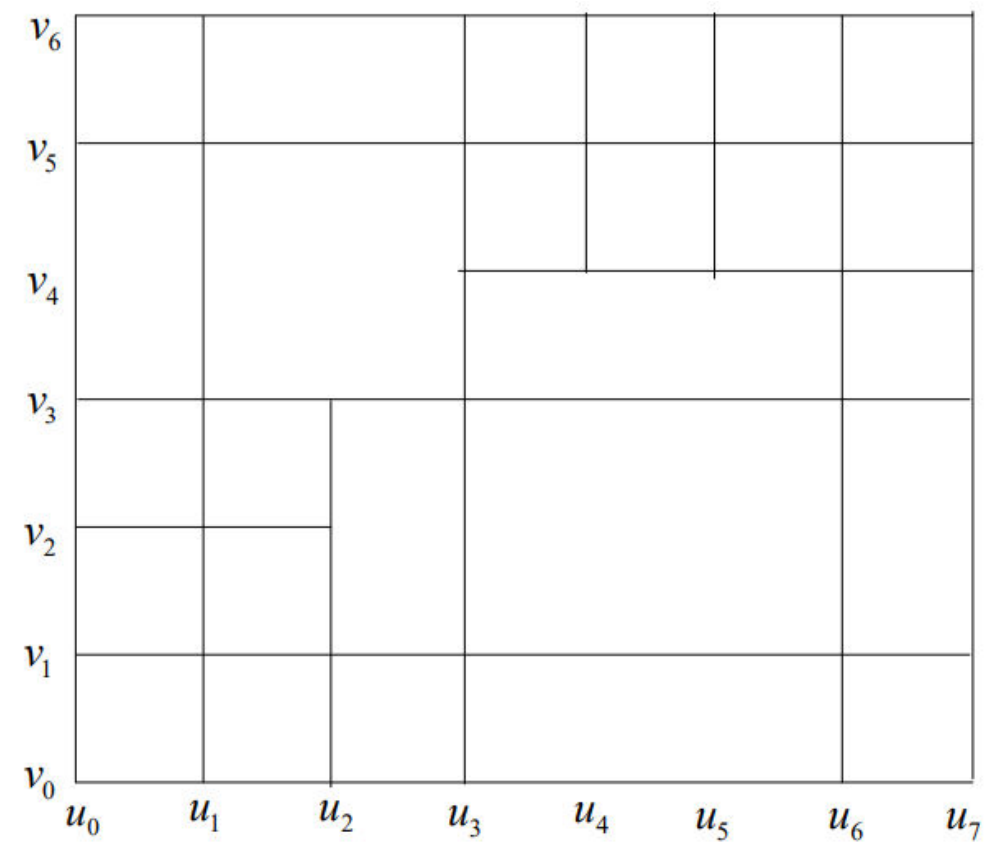

Figure 7. The second example. 
Example 3. For any $T$-mesh with knot vectors, $\left\{u_{i}\right\}$ where $u_{i} \neq u_{i+1}$, and $\left\{v_{j}\right\}$, where $v_{j} \neq v_{j+1}$, (see Figure 8), the T-splines always form a set of linearly independent functions of degree one.

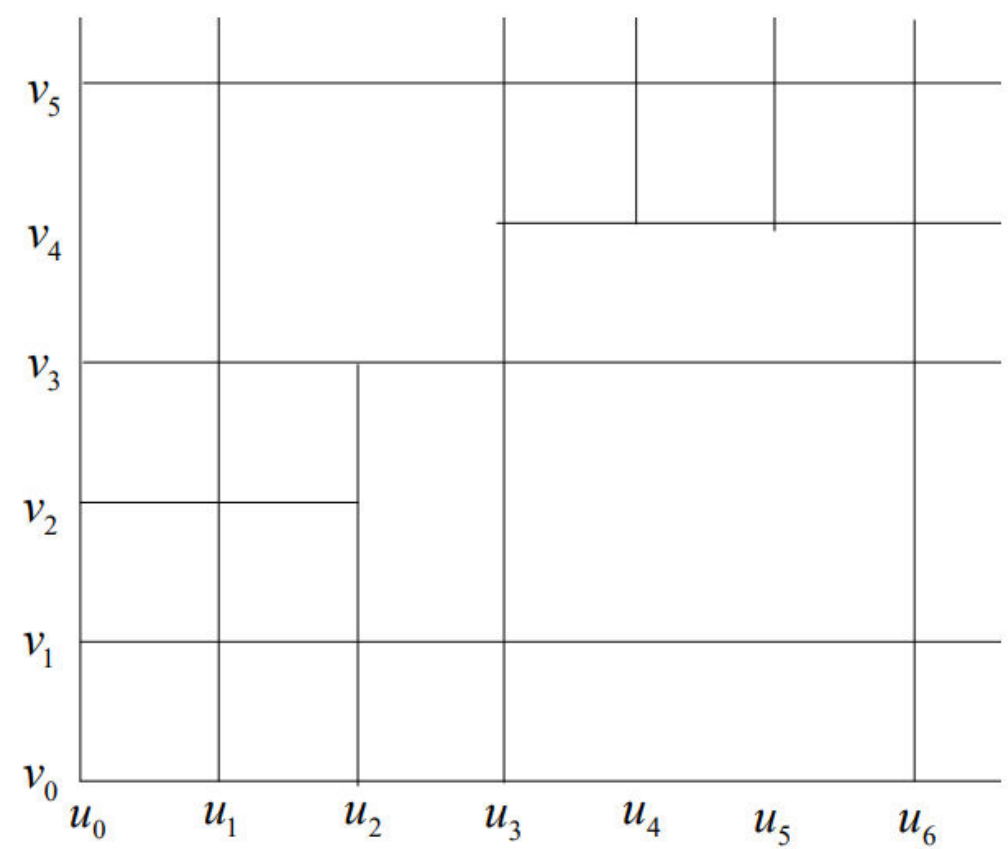

Figure 8. The third example.

The advantage of the proposed approach is that, with the result of linear independence, we can skip judging whether blending functions are analysis-suitable or not in IGA. From one point of view, the judging process is a troublesome problem existing in blending functions of higher degree, because some T-splines may have linearly dependent blending functions and cannot be used in IGA (see Figure 9). However, this analysis problem can be avoided by T-splines of degree one in this paper.

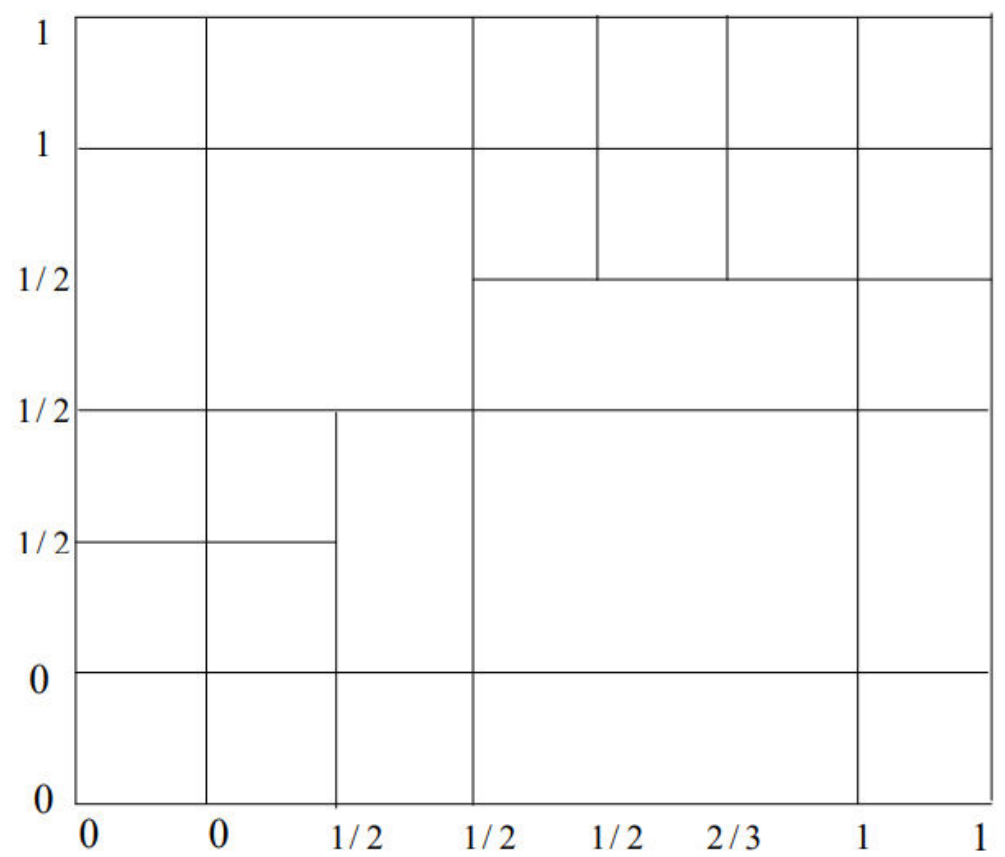

Figure 9. A linearly dependent T-spline of degree three [9]. 


\section{Isogeometric Analysis Example}

A significant difference between FEA and IGA is that IGA is based on the spline basis, which can represent the exact model geometrically and eliminate the error from the origin. The flow chart of isogeometric analysis using T-splines is shown in Figure 10, where $\mathrm{K}^{\mathrm{e}}$ and $\mathrm{F}^{\mathrm{e}}$ represent the element stiffness matrix and force vector, respectively, while $\mathrm{K}$ and F represent the global stiffness matrix and force vector, respectively. From Figure 10, the main process is divided into the following four parts: building IGA mesh connectivity, introducing boundary conditions, calculating global stiffness matrix and force vector, and calculating displacement and stress.

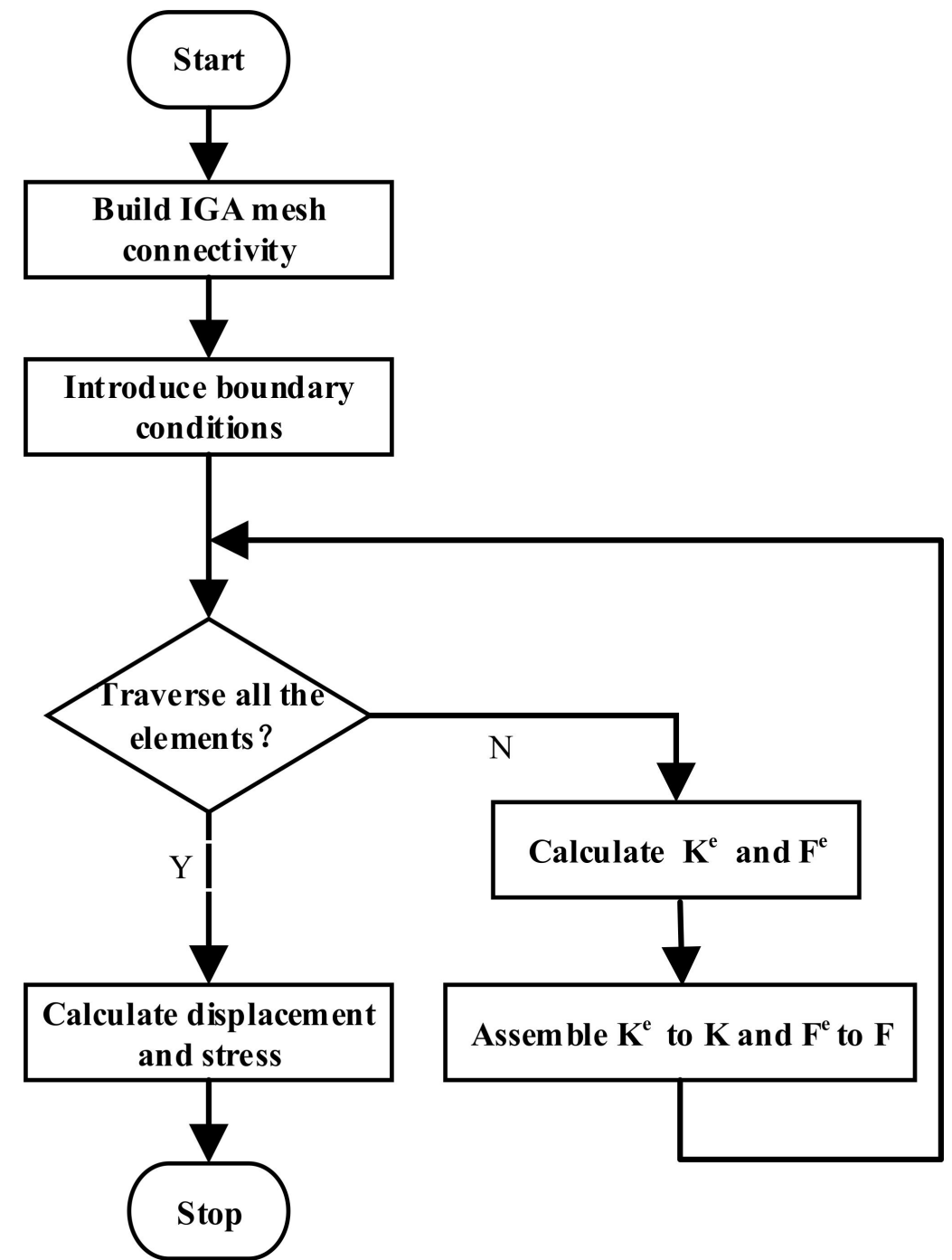

Figure 10. Isogeometric analysis using T-splines of degree one.

In this section, an isogeometric analysis example of elasticity is also presented. As Figure 11 shows, a wrench's jaw is fixed and the A, B points are subjected to forces $\mathrm{F}_{\mathrm{A}}=\mathrm{F}_{\mathrm{B}}=1.0 \mathrm{~N}$. The wrench's force analysis can be reduced to a two-dimensional problem. Degree one T-spline is used to build the wrench's model and the T-mesh of this model is shown in Figure 12. Here, we convert the degree one T-spline model into degree one Bézier model and do the IGA routines. For this model we just show that such a process is effective. 


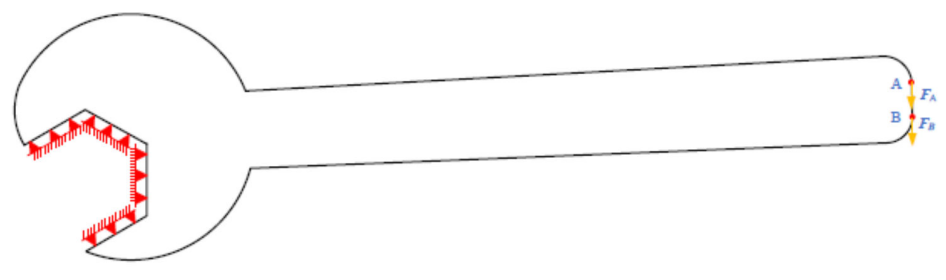

Figure 11. The problem description.

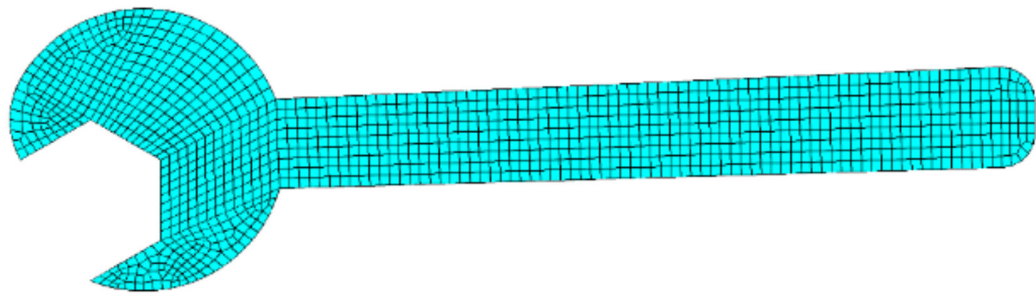

Figure 12. T-mesh of the wrench model.

Figure 13 shows a results comparison of displacement and Mises stress based on FEA and IGA. FEA results, acquired from Abaqus, are used as a benchmark for the capabilities of IGA. As given in (a)-(b) and (c)-(d), x-displacement and y-displacement magnitudes are rendered consistently between two methods and the maximum displacements occur at the end of the handle. Mises stress distributions of two analysis plotted in (e)-(f) also agrees well, and the maximum stresses appear at the boundary between the jaw and handle of the wrench model.

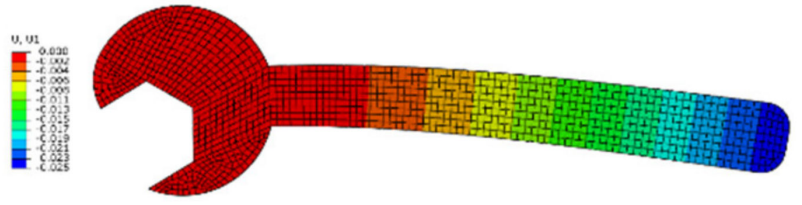

(a)

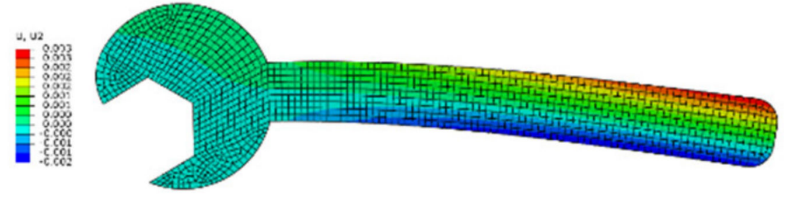

(c)

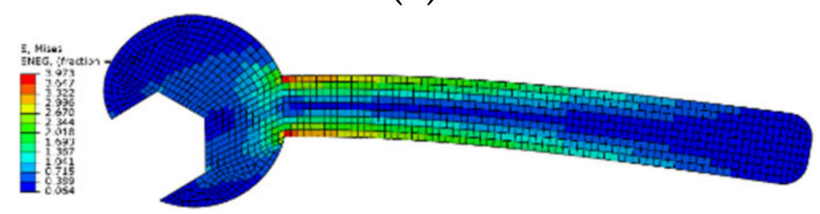

(e)

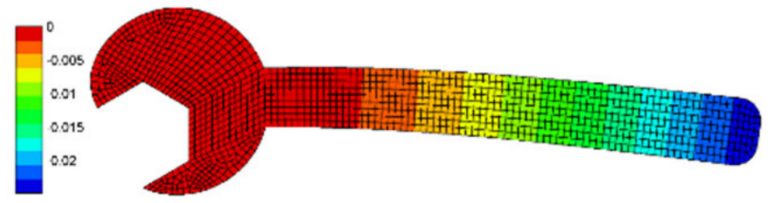

(b)

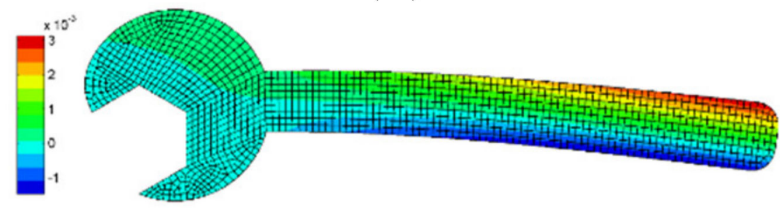

(d)

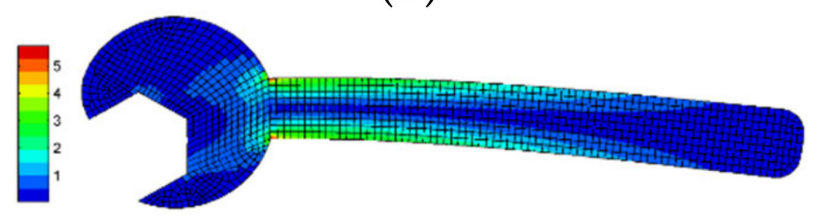

(f)

Figure 13. (a) x-displacement by Abaqus; (b) x-displacement by IGA; (c) y-displacement by Abaqus; (d) y-displacement by IGA; (e) Mises stress by Abaqus; (f) Mises stress by IGA.

The results give a powerful demonstration that IGA based on T-spline of degree one is comparable to FEA. Due to linear independence of its blending functions, T-spline of degree one can be used in IGA directly without considering any analysis-suitable conditions. So T-spline of degree one may have a flourish application on IGA of merging multi-patches and adjusting analysis results. 


\section{Conclusions and Future Work}

The results in this paper provide a mathematical foundation of one degree T-splines for isogeometric analysis. Though practical application, a three or more than three degree T-spline is likely to be used for geometry modeling as well as isogeometric analysis, the one degree T-spline model can avoid the problem of judging whether the model is analysissuitable or not. This may provide a new way of using T-spline for a CAD and CAE integrating scenario, since the one degree T-spline still guarantees the topology flexibility and is compatible with the spline-based modeling system. Especially for occasions that need a quick response from the analysis results, the one degree T-spline can exert its functions for its simplicity. Future work will analyze T-splines of high degrees that have linearly independent blending functions with more general topology.

Author Contributions: Conceptualization, A.W.; methodology, A.W.; writing-original draft, A.W., L.L., W.W., X.D., F.X., Z.C. and G.Z.; writing—review and editing, A.W., L.L. and G.Z. All authors have read and agreed to the published version of the manuscript.

Funding: This work was supported by opening fund of State Key Laboratory of Lunar and Planetary Sciences (Macau University of Science and Technology) (Macau FDCT grant No. 119/2017/ A3), 2017 Special Scientific Research on Civil Aircraft Project, and the Natural Science Foundation of China (Project No. 61972011 and 61572056).

Conflicts of Interest: The authors declare no conflict of interest.

\section{References}

1. Hughes, T.J.R.; Cottrell, J.A.; Bazilevs, Y. Isogeometric analysis: CAD, finite elements, NURBS, exact geometry, and mesh refinement. Comput. Methods Appl. Mech. Eng. 2005, 194, 4135-4195. [CrossRef]

2. Cottrell, J.A.; Hughes, T.J.R.; Bazilev, Y. Isogeometric Analysis: Toward Integration of CAD and FEA; Wiley: Chichester, UK, 2009.

3. Cottrell, J.A.; Hughes, T.J.R.; Reali, A. Studies of refinement and continuity in isogeometric structural analysis. Comput. Methods Appl. Mech. Eng. 2007, 196, 4160-4183. [CrossRef]

4. Bazilevs, Y.; Calo, V.; Cottrell, J.; Evans, J.; Hughes, T.J.R.; Lipton, S.; Scott, M.; Sederberg, T.W. Isogeometric analysis using T-splines. Comput. Methods Appl. Mech. Eng. 2010, 199, 229-263. [CrossRef]

5. Sederberg, T.W.; Zheng, J.; Bakenov, A.; Nasri, A. T-splines and T-NURCCs. ACM Trans. Graph. 2003, 22, 477-484. [CrossRef]

6. Sederberg, T.W.; Cardon, D.L.; Finnigan, G.T.; North, N.S.; Zheng, J.; Lyche, T. T-spline simplification and local refinement. ACM Trans. Graph. 2004, 23, 276-283. [CrossRef]

7. Vázquez, R.; Buffa, A. Isogeometric analysis for electromagnetic problems. IEEE Trans. Magn. 2010, 46, 3305-3308. [CrossRef]

8. Da Veiga, L.B.; Buffa, A.; Rivas, J.; Sangalli, G. Some estimates for h-p-k-refinement in Isogeometric Analysis. Numer. Math. 2010, 118, 271-305. [CrossRef]

9. Buffa, A.; Cho, D.; Sangalli, G. Linear independence of the T-spline blending functions associated with some particular T-meshes. Comput. Methods Appl. Mech. Eng. 2010, 199, 1437-1445. [CrossRef]

10. Wang, A.; Zhao, G. The Analysis of T-spline Blending Functions Linear Independence. Comput. Aided Des. Appl. 2011, 8, 735-745.

11. Li, X.; Zheng, J.; Sederberg, T.W.; Hughes, T.J.R.; Scott, M.A. On linear independence of T-spline blending functions. Comput. Aided Geomet. Des. 2012, 29, 63-76. [CrossRef]

12. Li, X.; Zhang, J. AS++ T-splines: Linear independence and approximation. Comput. Methods Appl. Mech. Eng. 2018, 333, $462-474$. [CrossRef]

13. Phung-Van, P.; Thanh, C.L.; Nguyen-Xuan, H.; Abdel-Wahab, M. Nonlinear transient isogeometric analysis of FG-CNTRC nanoplates in thermal environments. Compos. Struct. 2018, 20, 882-892. [CrossRef]

14. Hsu, M.-C.; Akkerman, I.; Bazilevs, Y. High-performance computing of wind turbine aerodynamics using isogeometric analysis. Comput. Fluid 2011, 49, 93-100. [CrossRef]

15. Grossmann, D.; Jüttler, B.; Schlusnus, H.; Barner, J; Vuong, A.V. Isogeometric simulation of turbine blades for aircraft engines. Comput. Aided Geom Des. 2012, 29, 519-531. [CrossRef]

16. Phung-Van, P.; Thai, C.H.; Nguyen-Xuan, H.; Wahab, M.A. Porosity-dependent nonlinear transient responses of functionally graded nanoplates using isogeometric analysis. Compos. Part B Eng. 2019, 164, 215-225. [CrossRef]

17. Phung-Van, P.; Lieu, Q.X.; Nguyen-Xuan, H.; Wahab, M.A. Size-dependent isogeometric analysis of functionally graded carbon nanotube-reinforced composite nanoplates. Compos. Struct. 2017, 166, 120-135. [CrossRef]

18. Thanh, C.L.; Tran, L.V.; Bui, T.Q.; Nguyen, H.X.; Abdel-Wahab, M. Isogeometric analysis for size-dependent nonlinear thermal stability of porous FG microplates. Compos. Struct. 2019, 221, 110838. [CrossRef]

19. Hao, P.; Wang, Y.; Ma, R.; Liu, H.; Wang, B.; Li, G. A new reliability-based design optimization framework using isogeometric analysis. Comput. Methods Appl. Mech. Eng. 2019, 345, 476-501. [CrossRef] 
20. Kwessi, E.; de Souza, G.; Djitte, N.; Ndiaye, M. The special atom space and Haar wavelets in higher dimensions. Demonstr. Math. 2020, 53, 131-151. [CrossRef]

21. Hughes, T.J.R. The Finite Element Method: Linear Static and Dynamic Finite Element Analysis; Dover Publications: Mineola, NY, USA, 2000. 